\title{
Coupling a Single Trapped Atom to a Whispering-Gallery-Mode Microresonator
}

\author{
Elisa Will@ ${ }^{1}$ Luke Masters $\odot,{ }^{1,2}$ Arno Rauschenbeutel® ${ }^{1,2}$ Michael Scheucher $\odot,{ }^{1}$ and Jürgen Volz ${ }^{1,2, *}$ \\ ${ }^{1}$ Vienna Center for Quantum Science and Technology, Atominstitut, TU Wien, 1020 Vienna, Austria \\ ${ }^{2}$ Department of Physics, Humboldt-Universität zu Berlin, 10099 Berlin, Germany
}

(Received 12 February 2021; accepted 29 April 2021; published 11 June 2021)

\begin{abstract}
We demonstrate trapping of a single ${ }^{85} \mathrm{Rb}$ atom at a distance of about $200 \mathrm{~nm}$ from the surface of a whispering-gallery-mode bottle microresonator. The atom is trapped in an optical potential, which is created by retroreflecting a red-detuned focused laser beam from the resonator surface. We counteract the trap-induced light shift of the atomic transition frequency by superposing a second laser beam. This allows us to observe a vacuum Rabi splitting in the excitation spectrum of the coupled atom-resonator system. This first demonstration of stable and controlled interaction of a single atom with a whispering-gallery mode in the strong coupling regime opens up the route toward the implementation of quantum protocols and applications that harvest the chiral atom-light coupling present in this class of resonators.
\end{abstract}

DOI: 10.1103/PhysRevLett.126.233602

In free space, the interaction between single atoms and single photons is weak. However, by strongly confining the photons inside a microresonator with high quality factor $Q$, and by coupling the atoms to the resonator mode, atom-light interaction can be significantly enhanced. This approach lies at the heart of cavity quantum electrodynamics (CQED) [1-3]. Over the past decades, ultrahigh finesse Fabry-Perot microresonators have been instrumental in advancing this field. In these traditional resonators, single atoms have been trapped inside the resonator mode $[4,5]$, which has led to many ground-breaking experiments [6].

More recently, other resonator types have successfully been employed in single-atom CQED, such as optical fiber-based Fabry-Perot cavities [7-10], photonic crystal cavities [11,12], optical nanofiber-based cavities [13,14], and whispering-gallery-mode (WGM) microresonators [15-17]. Out of those, WGM resonators distinguish themselves by offering chiral, i.e., propagation-directiondependent, light-matter interaction [16,18], which enables novel protocols and functionalities for processing light on the quantum level [17,19-21]. However, so far, only freefalling atoms have been coupled to WGM resonators, resulting in probabilistic operation, a limited interaction time, and a position-dependent coupling strength, which reduces process fidelities. Gaining the ability to trap atoms inside the resonator field is the crucial prerequisite for overcoming the current limitations and harnessing the full potential of this class of resonators for future applications.

Published by the American Physical Society under the terms of the Creative Commons Attribution 4.0 International license. Further distribution of this work must maintain attribution to the author(s) and the published article's title, journal citation, and DOI.
Here, we demonstrate strong coupling of a single trapped rubidium atom to a WGM bottle microresonator with ultrahigh quality factor $Q$ [22]. We achieve this by overcoming two crucial experimental challenges. First, efficient coupling to the evanescent field of the WGM requires the atom to be trapped at a small distance from the resonator surface where van der Waals forces and Casimir-Polder forces have to be counteracted. To achieve this, we employ a deep standing-wave optical dipole trap, created by retroreflecting a focused trapping light field from the resonator surface [11]. Second, the intense light field of the trap induces a substantial light shift, which leads to a position-dependent detuning of the atomic resonance from the resonator mode. We counteract this light shift of the atomic transition by means of a second, detuned compensation light field that shifts the excited state back into resonance [23]. As a result, we observe a resonant vacuum Rabi splitting in the excitation spectrum of the trapped atom-resonator system, indicating strong coupling.

The core elements of the experimental setup are shown in Fig. 1(a). The WGM bottle microresonator features a quality factor of $Q \approx 5 \times 10^{7}$ and is stabilized to the resonance of the unperturbed $\left(5 S_{1 / 2}, F=3\right) \rightarrow$ $\left(5 P_{3 / 2}, F^{\prime}=4\right)$ transition of ${ }^{85} \mathrm{Rb}$ with angular frequency $\omega_{0}$. To couple light into and out of the resonator, it is interfaced with a tapered fiber coupler. The system is set to critical coupling such that, in the absence of a coupled atom, the transmission of resonant light through the coupling fiber vanishes. This occurs when the fiberresonator coupling rate $\kappa_{\mathrm{ext}}$ equals the intrinsic resonator field decay rate $\kappa_{0}$. The total resonator field decay rate is then given by $\kappa=\kappa_{0}+\kappa_{\mathrm{ext}} \approx 2 \pi \times 10 \mathrm{MHz}$.

The optical dipole trap is created by retroreflecting a focused laser beam (waist radius: $w_{\text {trap }}=3.5 \pm 0.3 \mu \mathrm{m}$ ) from the bottle resonator surface. The wavelength of the 


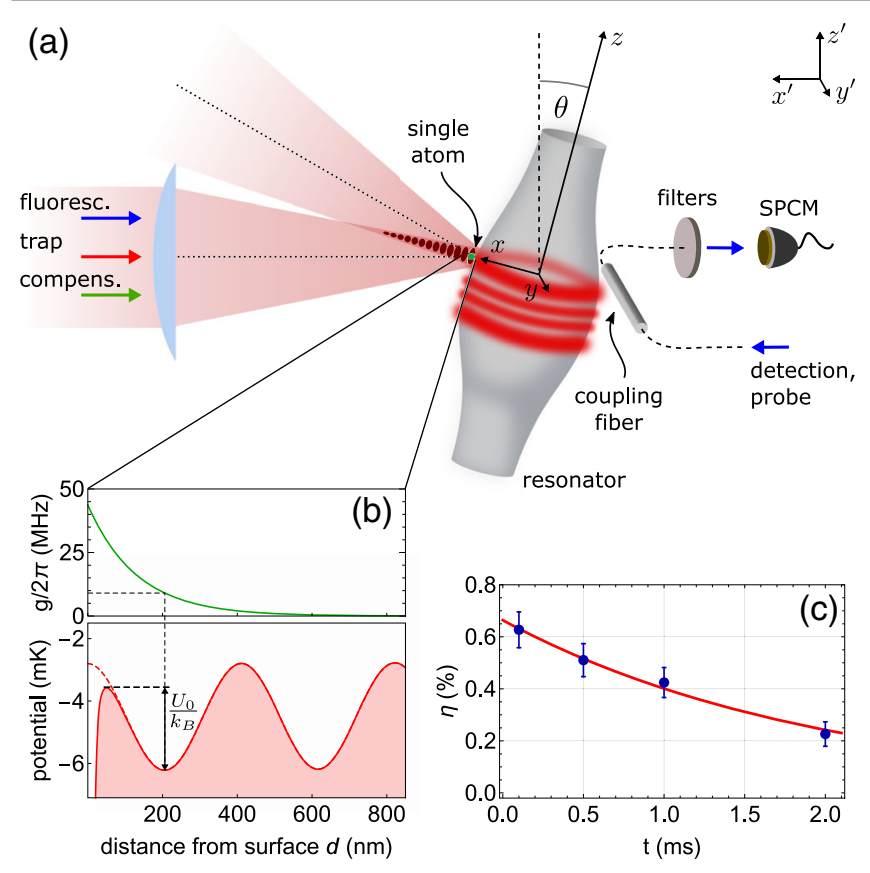

FIG. 1. (a) Experimental setup for detecting and trapping a single ${ }^{85} \mathrm{Rb}$ atom close to a bottle microresonator in a standingwave optical dipole trap. The optics for the dipole trap is also used to focus a laser beam onto the atom for fluorescence detection, as well as a laser beam for light shift compensation (see main text). The resonator is tilted by $\theta \approx 17^{\circ}$. Therefore, we define a resonator-centered coordinate system $(x, y, z)$ and a laboratory-centered coordinate system $\left(x^{\prime}, y^{\prime}=y, z^{\prime}\right)$. (b) Trap potential (bottom panel) and expected coupling strength, $g$ (top panel), along the $x$ direction. The steep drop of the potential toward the resonator surface is caused by attractive surface forces. (c) Measurement of the probability for finding a given detected single atom in the trap as a function of the waiting time $t$. The red line is an exponential fit, yielding a trap lifetime of $\tau \approx 2 \mathrm{~ms}$.

trapping light $\left(\lambda_{\text {trap }}=783.68 \mathrm{~nm}\right)$ is red detuned with respect to the $5 S_{1 / 2} \rightarrow 5 P_{3 / 2}$ transition, see Fig. 2 . The interference between the incident beam and its reflection forms a partially modulated standing-wave pattern along the $x$ axis. Figure 1(b) shows the trap potential and the expected atom-light coupling strength as a function of the distance $d$ of the atom from the resonator surface in the $x$ direction. We trap a single atom in the potential minimum closest to the resonator, which is located at $\lambda_{\text {trap }} /(4 \cos \theta) \approx 205 \mathrm{~nm}$. For this position, we expect an atom-light coupling strength of $g \approx 2 \pi \times 10 \mathrm{MHz}$ which puts the system at the onset of strong coupling, i.e., $g>(\kappa, \gamma)$, where $\gamma=2 \pi \times 3 \mathrm{MHz}$ is the atomic dipole decay rate.

The trap is loaded from a 1-cm diameter cloud of about $5 \times 10^{7}$ laser-cooled ${ }^{85} \mathrm{Rb}$ atoms that is delivered to the resonator by an atomic fountain and has a temperature of about $7 \mu \mathrm{K}$. In order to detect the presence of a single atom in the resonator mode in real time, we send resonant detection light through the coupling fiber and monitor the

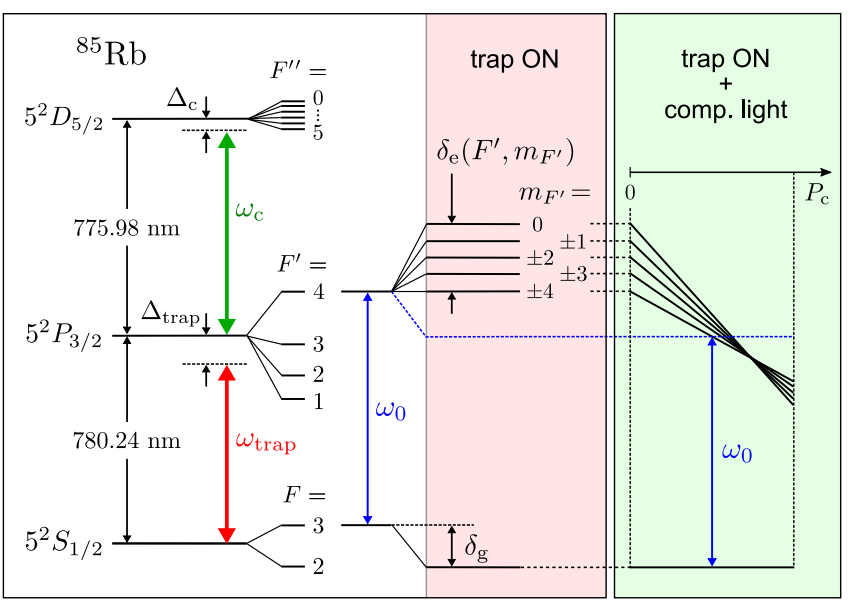

FIG. 2. Principle of the light shift compensation. The left box shows the energy level structure of ${ }^{85} \mathrm{Rb}$ relevant for the compensation scheme and illustrates the trap-induced light shifts of the $\left(5 S_{1 / 2}, F=3\right) \rightarrow\left(5 P_{3 / 2}, F^{\prime}=4\right)$ atomic transition, for a fixed power $P_{\text {trap. }}$ The right box qualitatively shows how the $F^{\prime}=4$ Zeeman levels are tuned across resonance, when the compensation laser power $P_{c}$ is increased in the presence of the trap light. A quantitative treatment of the compensation mechanism is shown in Fig. 3(a).

transmitted power using a single-photon counting module (SPCM). The atom-resonator interaction leads to a transmission increase, to which we react using a field programmable gate array-based detection and control system [16]. Upon detection of an atom, the detection light is switched off using an electro-optical modulator, and the dipole trap is switched on using an acousto-optical modulator. The overall delay between detection and trapping is about $250 \mathrm{~ns}$. As this duration is much shorter than the average transit time of an atom through the evanescent field of the resonator mode, it allows us to catch a detected atom if it is located inside the trapping volume.

In order to verify that the trap loading succeeded, we use a fluorescence detection scheme. We launch a $20 \mu$ s-long detection light pulse through the trap optics onto the atom, which has a center frequency of $\omega_{0}$ and a peak intensity of about $100 I_{\text {sat }}$. Here, $I_{\text {sat }}$ is the saturation intensity of the $\left(5 S_{1 / 2}, F=3\right) \rightarrow\left(5 P_{3 / 2}, F^{\prime}=4\right)$ transition. A fraction of the scattered fluorescence photons is coupled into the resonator mode and detected with the SPCM, see Fig. 1(a). This allows us to detect the presence of a resonator-coupled atom in the dipole trap despite the large trap-induced atom-resonator detuning, see below. By varying the timing of the detection light pulse, we measure the probability for finding a single atom at a waiting time $t$ after switching on the trap, see Fig. 1(c). Fitting an exponential function (red solid line) to the measured probabilities, $\eta(t)=\eta_{0} \exp (-t / \tau)$, yields a probability for trapping a given detected single atom of $\eta_{0} \approx 0.7 \%$ and a trap lifetime of $\tau \approx 2 \mathrm{~ms}$. This trapping probability matches our expectation considering the finite overlap of the trap volume with 
the resonator mode, the initial kinetic energy of the atom and the time delay between atom detection and switching on the trap.

In the moment of detection, the free-falling atoms have a mean kinetic energy corresponding to $E / k_{B} \approx 0.5 \mathrm{mK}$. Consequently, trapping an atom requires an optical potential with significant depth. We use a linearly polarized dipole trap beam with a power of $P_{\text {trap }}=19 \mathrm{~mW}$. For the trap light field polarized along the $y$ axis $\left(z^{\prime}\right.$ axis), the trap depth amounts to $U_{0} / k_{B} \approx 2.7 \mathrm{mK}\left(U_{0} / k_{B} \approx 1.9 \mathrm{mK}\right)$ with the corresponding axial and transverse trap frequencies $\left\{\omega_{x}, \omega_{y, z}\right\}=2 \pi \times\{1.00 \mathrm{MHz}, 71 \mathrm{kHz}\}\left(\left\{\omega_{x}, \omega_{y, z}\right\}=2 \pi \times\right.$ $\{0.86 \mathrm{MHz}, 68 \mathrm{kHz}\}$ ).

The trap light field induces a scalar light shift $\delta_{g}$ of the $5 S_{1 / 2}$ ground state and a scalar as well as tensor light shifts, $\delta_{e}\left(F^{\prime}, m_{F^{\prime}}\right)$, of the $5 P_{3 / 2}$ excited state, see Fig. 2. The resulting shifts of the $5 S_{1 / 2} \rightarrow 5 P_{3 / 2}$ transition frequencies, which depend both on the Zeeman state and the position of the atom in the trap, reach up to about $250 \mathrm{MHz}$. The corresponding effective broadening of the atomic transition exceeds by far the atomic and the resonator linewidths.

In order to efficiently interface the trapped atom with the resonator in the presence of this light shift, we have to compensate for the latter. For this purpose, we expose the atom to an additional light field-the so-called compensation field. Its polarization is aligned with the linear polarization of the trap field and its frequency is offset locked with a red detuning of $\Delta_{c} \approx 2 \pi \times 930 \mathrm{MHz}$ to the $5 P_{3 / 2} \rightarrow 5 D_{5 / 2}$ transition using absorption spectroscopy [24], see Fig. 2 and Supplemental Material [25]. The compensation field induces a scalar and tensor light shift on the $5 P_{3 / 2}$ excited state while its effect on the ground state is negligible.

For optimal compensation of the position-dependent trap-induced light shift of the $5 S_{1 / 2} \rightarrow 5 P_{3 / 2}$ transition, the intensity distribution of the compensation field should match that of the trapping field. This is approximately realized in our case, as the two light fields have similar wavelengths $\left(\lambda_{\text {trap }}=783.68 \mathrm{~nm}\right.$ and $\left.\lambda_{c}=775.98 \mathrm{~nm}\right)$. In our setup, we simultaneously send the two fields through the same fiber-coupled focusing optics, such that the positions of their foci, their waist radii, and their standing-wave patterns near the resonator closely match upon reflection off the resonator. This allows us to cancel the trap-induced scalar light shift of the transition in the entire trapping volume, when the power of the compensation field $P_{c}$ is adequately chosen.

Figure 3(a) shows the calculated detunings of the two-color light-shifted transition frequencies between the $5 S_{1 / 2}, F=3$ ground state and the $5 P_{3 / 2}$ excited state manifold as a function of $P_{c}$, see Supplemental Material [25]. Here, we take the frequency of the $F=3 \rightarrow F^{\prime}=4$ cycling transition in the absence of light shifts, $\omega_{0}$, as the reference, and we assume that the atom is located in the trap center. With increasing power of the compensation field,
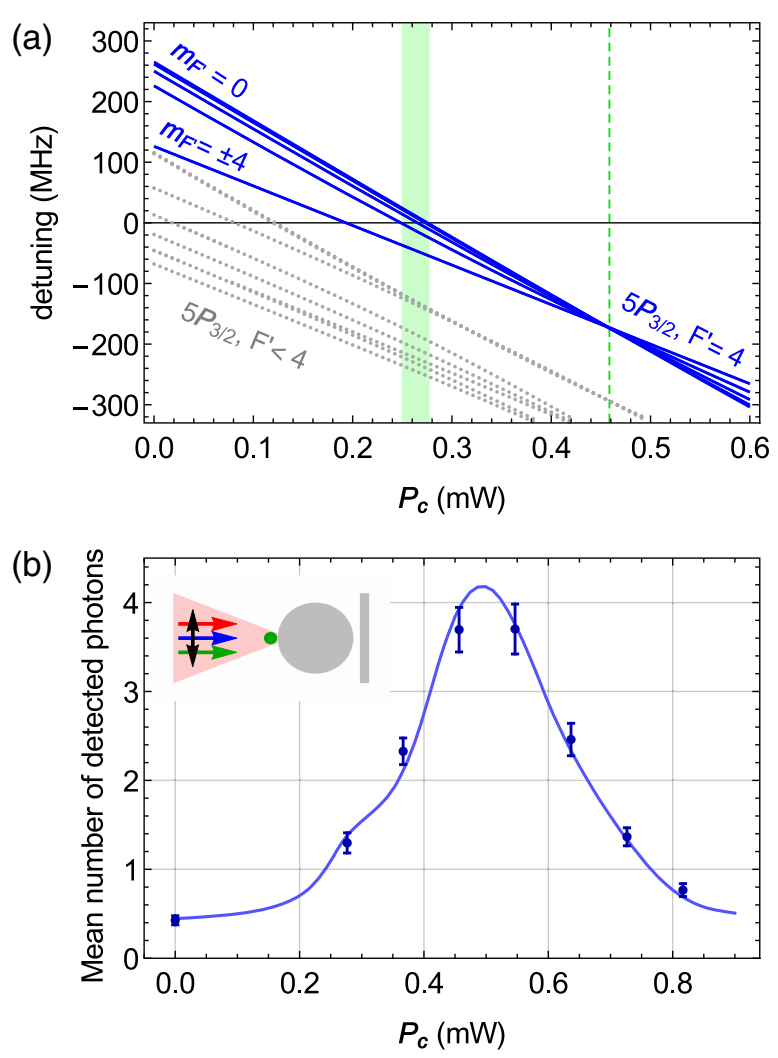

FIG. 3. (a) Calculation of the detunings of the two-color lightshifted transition frequencies between the $5 S_{1 / 2}, F=3$ ground state and the $5 P_{3 / 2}$ excited state manifold with respect to $\omega_{0}$, as a function of the compensation laser power $P_{c}$ for $y$-polarized light. For $z^{\prime}$-polarized light, the light shifts are about $10 \%$ smaller. The calculation is performed for the trap and compensation laser parameters given in the text and for an atom located at the trap center. The gray dotted lines are the detunings for the lower lying $F^{\prime}$ levels. (b) Measurement of the mean number of detected fluorescence photons in the presence of the trap and compensation lasers, as a function of the compensation laser power $P_{c}$.

both the scalar and tensor light shifts of the transition frequencies decrease. Within the range $P_{c}=0.250 \mathrm{~mW}$ to $0.276 \mathrm{~mW}$, the detunings of the transitions to the $\left(F^{\prime}=4,\left|m_{F^{\prime}}\right| \leq 3\right)$ excited states sequentially cross zero, see green shaded area. The detuning of the transitions to the $\left(F^{\prime}=4,\left|m_{F^{\prime}}\right|=4\right)$ levels vanishes at $\approx 0.197 \mathrm{~mW}$. For these powers, the light shifts of the ground state and the respective excited states are equal, see Fig. 2.

In order to experimentally find the point of optimal compensation we measure the fluorescence of a single trapped atom as a function of the compensation laser power. For this purpose, we send probe light resonant with the unperturbed $F=3 \rightarrow F^{\prime}=4$ transition through the trap optics for $100 \mu \mathrm{s}$ and measure the number of photons scattered into the resonator mode. In this measurement, the probe laser intensity was about $2 I_{\mathrm{sat}}$ at the center of the trap. The linear polarizations of the trap, the compensation, 
and the probe light fields were aligned along the $y$ axis. Figure 3(b) shows the mean number of fluorescence photons detected via the coupling fiber. Comparing this result with the calculation in Fig. 3(a) (green shaded area), reveals a shift of the expected resonance toward higher compensation laser powers. This is most likely caused by chromatic aberrations of our trap optics, which displace the foci of the trap and compensation laser beams with respect to each other along the beam axis. This results in a mismatch of their beam radii at the position of the atom. The blue solid line is a theoretical fit where we average fluorescence spectra for transitions between all Zeeman levels of the $F=3$ and $F^{\prime}=4$ manifolds. Here, we consider the position dependence of the two-color light shifts and, additionally, the occurrence of vector light shifts to take into account residual circular polarization components in the trap and compensation light fields. We average the calculated spectra over the position distribution of the atoms in the trap, which we estimate from an independent temperature measurement, see Supplemental Material [25]. We fit this average spectrum to the data using as fit parameters the beam radius mismatch, the maximum mean number of detected photons and the polarization ellipticity. This fit yields a ratio of the beam radii of $w_{c} / w_{\text {trap }}=1.5$ and a reduced overlap with linear polarization of about $96 \%$. We obtain a maximum scattering rate at a compensation laser power of $P_{c}^{\max }=500 \pm 50 \mu \mathrm{W}$. These results lie within the expectations for our experimental setup, and the theoretical fit agrees well with the experimental data.

We now investigate the atom-resonator interaction in the presence and absence of light shift compensation by measuring transmission spectra of the atom-resonator system. For this purpose, we align the linear polarizations of the dipole trap and the compensation light along the $z^{\prime}$ axis. With respect to this axis, the transverse-magnetic WGMs are approximately $\sigma^{+}$or $\sigma^{-}$polarized. The initial atom detection prepares the atom in the outermost Zeeman level $F=3, m_{F}=3$. Thus, when probing the $\sigma^{+}$-polarized WGM, the atom behaves approximately as a two-level system, where the $\left(F=3, m_{F}=3\right) \rightarrow\left(F^{\prime}=4, m_{F^{\prime}}=4\right)$ cycling transition interacts exclusively with the probed resonator mode [16]. The cycling transition features a smaller light shift compared to the transitions to the other Zeeman levels of the $F^{\prime}=4$ excited state. Thus, we expect resonant atom-resonator interaction for a compensation laser power of $\sim 0.8 P_{c}^{\max }$, see Fig. 3(a).

We measure the transmission spectra of the coupled atom-resonator system by launching probe light through the coupling fiber into the $\sigma^{+}$-polarized resonator mode. We vary the probe-resonator detuning $\Delta \omega=\omega_{\text {probe }}-\omega_{0}$ and for each value we record the transmission, which is averaged over $400 \mu \mathrm{s}$. For a compensation laser power of $P_{c}^{\exp }=330 \pm 30 \mu \mathrm{W}$ we measure a spectrum with an almost symmetric vacuum Rabi splitting, see Fig. 4(a). The solid line is the theoretical prediction for the symmetric

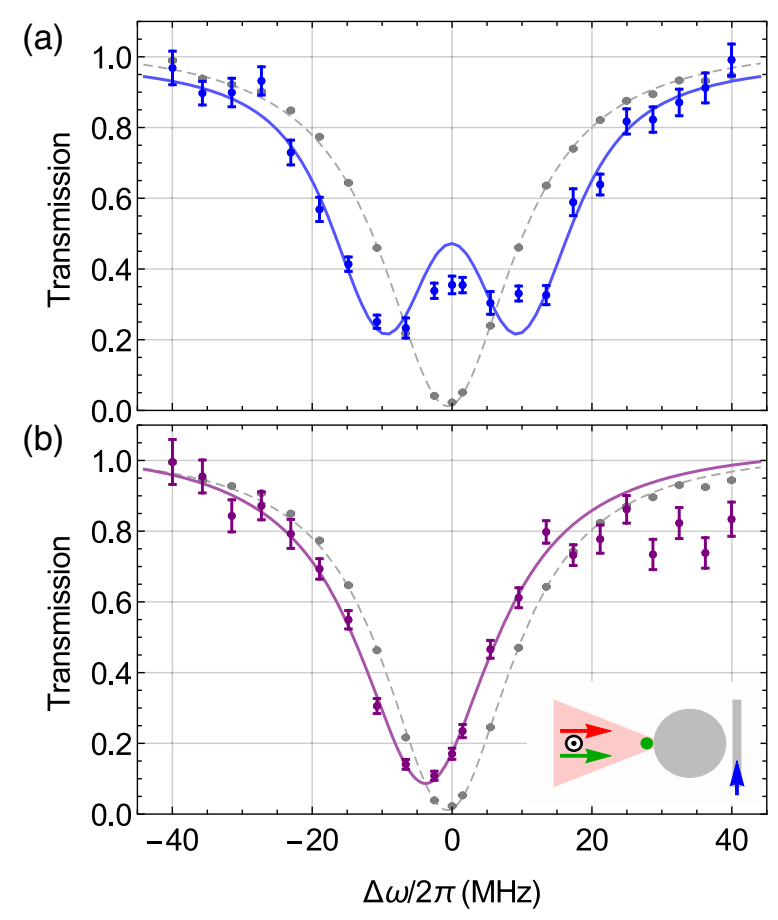

FIG. 4. Normalized transmission spectra of the atom-resonator system (a) in presence $\left(P_{c}=330 \pm 30 \mu \mathrm{W}\right)$ and (b) absence of the compensation laser. Each plot shows the average spectrum of single trapped atoms (blue and purple, respectively), as well as the empty resonator spectrum when no atom was trapped (gray). The error bars of the gray data points are smaller than the point size. The solid line in (a) is a theoretical prediction (see details in the text). The solid line in (b) is a fit of a Lorentzian function to the data, excluding the six rightmost data points, as a guide to the eye. The dashed lines are fitted Lorentzians with a HWHM of about $11 \mathrm{MHz}$. The inset in (b) illustrates the propagation directions of the light fields, and the polarization direction of the trap and compensation fields.

vacuum Rabi spectrum, obtained for a compensation laser power of $P_{c}^{\text {th }}=400 \mu \mathrm{W}$. The $17 \%$ discrepancy between $P_{c}^{\exp }$ and $P_{c}^{\text {th }}$ we mostly attribute to the error in determining $P_{c}^{\max }$ from Fig. 3(b). The predicted spectrum was calculated by averaging vacuum Rabi spectra over the positiondependent atom-photon coupling strengths and light shifts for the same position distribution and beam radius mismatch as the ones used for analyzing Fig. 3(b). The model agrees well with the measurement and yields an average coupling strength of $g^{\text {th }} / 2 \pi=9.3 \mathrm{MHz}$ with a standard deviation of $\sigma_{g}^{\text {th }} / 2 \pi=6.1 \mathrm{MHz}$, in good agreement with our expectations. This result demonstrates, for the first time, strong coupling of a single trapped atom to a WGM resonator. We note that the light shift compensation not only compensates the mean light shift of the atomic transition, but also reduces the substantial broadening of the latter and the concurrent blurring of the Rabi splitting due to the atomic motion in the trap. Figure 4(b) shows the transmission spectrum in the absence of light shift compensation. In this case, due to the large atom-resonator 
detunings, we only observe a small dispersive and dissipative effect of the atom.

In summary, we optically trapped a single atom at a distance of about $200 \mathrm{~nm}$ from the surface of a WGM microresonator and compensated the position-dependent trap-induced light shift of the atomic transition using a twocolor magic wavelength scheme. This allowed us to demonstrate stable and controlled interaction of a single atom with a whispering-gallery mode in the strong coupling regime, thereby overcoming a long-standing challenge of optical cavity quantum electrodynamics. The demonstrated method can also be applied to WGM and ring resonators on integrated optical chips. This lays the pathway toward realizing more complex quantumcontrolled photonic circuits, which may in particular profit from the chiral nature of atom-light coupling in this setting.

We thank Adèle Hilico for her contributions in the early stages of the experiment. This work was financially supported by the Austrian Science Fund (FWF; SFB FoQuS Project No. F 4017 and DK CoQuS Project No. W 1210-N16), the European Union's Horizon 2020 research and innovation programme under Grant Agreements No. 800942 and No. 899275, and the Alexander von Humboldt-Foundation (Alexander von Humboldt Professorship).

*juergen.volz@hu-berlin.de

[1] R. J. Thompson, G. Rempe, and H. J. Kimble, Phys. Rev. Lett. 68, 1132 (1992).

[2] M. Brune, F. Schmidt-Kaler, A. Maali, J. Dreyer, E. Hagley, J. M. Raimond, and S. Haroche, Phys. Rev. Lett. 76, 1800 (1996).

[3] A. Kuhn, M. Hennrich, and G. Rempe, Phys. Rev. Lett. 89, 067901 (2002).

[4] J. Ye, D. W. Vernooy, and H. J. Kimble, Phys. Rev. Lett. 83, 4987 (1999).

[5] P. W. H. Pinkse, T. Fischer, P. Maunz, and G. Rempe, Nature (London) 404, 365 (2000).

[6] A. Reiserer and G. Rempe, Rev. Mod. Phys. 87, 1379 (2015).

[7] J. Volz, R. Gehr, G. Dubois, J. Estève, and J. Reichel, Nature (London) 475, 210 (2011).

[8] M. Steiner, H. M. Meyer, C. Deutsch, J. Reichel, and M. Köhl, Phys. Rev. Lett. 110, 043003 (2013).

[9] J. Gallego, W. Alt, T. Macha, M. Martinez-Dorantes, D. Pandey, and D. Meschede, Phys. Rev. Lett. 121, 173603 (2018).
[10] M. Brekenfeld, D. Niemietz, J. D. Christesen, and G. Rempe, Nat. Phys. 16, 647 (2020).

[11] J. D. Thompson, T. G. Tiecke, N. P. de Leon, J. Feist, A. V. Akimov, M. Gullans, A. S. Zibrov, V. Vuletić, and M. D. Lukin, Science 340, 1202 (2013).

[12] A. Goban, C.-L. Hung, J. D. Hood, S.-P. Yu, J. A. Muniz, O. Painter, and H. J. Kimble, Phys. Rev. Lett. 115, 063601 (2015).

[13] S. Kato and T. Aoki, Phys. Rev. Lett. 115, 093603 (2015).

[14] K. P. Nayak, J. Wang, and J. Keloth, Phys. Rev. Lett. 123, 213602 (2019).

[15] T. Aoki, B. Dayan, E. Wilcut, W. P. Bowen, A. S. Parkins, T. J. Kippenberg, K. J. Vahala, and H. J. Kimble, Nature (London) 443, 671 (2006).

[16] C. Junge, D. O'Shea, J. Volz, and A. Rauschenbeutel, Phys. Rev. Lett. 110, 213604 (2013).

[17] I. Shomroni, S. Rosenblum, Y. Lovsky, O. Bechler, G. Guendelman, and B. Dayan, Science 345, 903 (2014).

[18] P. Lodahl, S. Mahmoodian, S. Stobbe, A. Rauschenbeutel, P. Schneeweiss, J. Volz, H. Pichler, and P. Zoller, Nature (London) 541, 473 (2017).

[19] D. O'Shea, C. Junge, J. Volz, and A. Rauschenbeutel, Phys. Rev. Lett. 111, 193601 (2013).

[20] M. Scheucher, A. Hilico, E. Will, J. Volz, and A. Rauschenbeutel, Science 354, 1577 (2016).

[21] O. Bechler, A. Borne, S. Rosenblum, G. Guendelman, O. E. Mor et al., Nat. Phys. 14, 996 (2018).

[22] M. Pöllinger, D. O'Shea, F. Warken, and A. Rauschenbeutel, Phys. Rev. Lett. 103, 053901 (2009).

[23] A. P. Hilton, C. Perrella, A. N. Luiten, and P. S. Light, Phys. Rev. Applied 11, 024065 (2019).

[24] A. Yu. Kalatskiy, A. E. Afanasiev, P. N. Melentiev, and V. I. Balykin, Laser Phys. 27, 055703 (2017).

[25] See Supplemental Material at http://link.aps.org/ supplemental/10.1103/PhysRevLett.126.233602 for a detailed description of the experimental methods and theoretical models as well as additional measurements, which includes Refs. [26-30].

[26] W. Alt, D. Schrader, S. Kuhr, M. Müller, V. Gomer, and D. Meschede, Phys. Rev. A 67, 033403 (2003).

[27] F. Le Kien, P. Schneeweiss, and A. Rauschenbeutel, Eur. Phys. J. D 67, 92 (2013).

[28] D. A. Steck, Rubidium 85 D line data, (revision 2.1.6, 20 September 2013), http://steck.us/alkalidata.

[29] H. J. Carmichael, Dissipation in quantum mechanics: The master equation approach, in Statistical Methods in Quantum Optics 1: Master Equations and Fokker-Planck Equations (Springer Berlin Heidelberg, Berlin, Heidelberg, 1999).

[30] Y. Louyer, D. Meschede, and A. Rauschenbeutel, Phys. Rev. A 72, 031801(R) (2005). 